\title{
A Call to Action for More Effective Preventative Care Strategies for HIV in Men Having Sex with Men (MSM): A Combined Computational Model and Qualitative Analysis
}

\section{James Yeongjun Park*}

Department of Physics, Emory University, USA

\begin{abstract}
While HIV is now considered a chronic and manageable disease in many developed countries largely attributable to advances in antiretroviral therapy (ART); there are unanswered questions regarding certain aspects of disease progression. The global literature consistently demonstrates that creating effective access to healthcare and antiretroviral therapy (ART), providing substance abuse prevention and treatment programs, and optimizing adherence to ART will improve HIV treatment outcomes. Nonetheless, psychosocial and cultural factors such as stigma and discrimination (as a consequence of being a sexual minority, HIV-positive, an active drug user), depression, and social isolation have been documented to have an adverse effect on HIV treatment. Macro-social determinants like access to healthcare and sociocultural like stigma and discrimination as well as psychosocial determinants like depression have an adverse effect on HIV outcomes such as ART adherence as well as overall health status or outcomes. In this study, I provide a framework incorporating all biomedical, behavioral, and structural interventions that comprehensively and systemically pose the dire need for more effective preventative care strategies for HIV in MSM. With the knowledge gained I posit that a clearer understanding of these issues among MSM living with HIV will emerge and guide further action along more specific directions. Also, I anticipate that these findings will permit a more precise quantification of each major risk factor's contribution to treatment outcome, thus informing policy and education efforts aimed at risk factor modification. This study points the way for future biologic, behavior, and structural interventions that could improve the quality of life for people living with HIV.
\end{abstract}

\section{Keywords: HIV; Sex; Antiretroviral therapy}

\section{Search Methods}

I searched electronic databases, current journals, manuscripts submitted by researchers, bibliographies of relevant articles, conference proceedings, and other reviews for published and unpublished reports from 1988 through January 2017. I also asked researchers at Emory University working in HIV prevention about new and ongoing studies. Studies were considered with equal weight if they examined the effects of behavioral and structural interventions aimed at reducing risk for HIV transmission among MSM. HIV incidences were reported in only a few studies so I did not perform quantitative analyses of these results. I systematically reviewed the HIV prevention literature to find studies introducing the effects of behavioral and structural interventions for MSM. Resources included online database, reviews and other studies in the HIV prevention literature, expert recommendation, hand searches of journals and manuscripts and unpublished reports submitted by researchers.

\section{Background}

The motivation of this study was elicited from Risk network structure in the early epidemic phase of HIV transmission in Colorado Springs by Potterat et al. [1]. I thank Dr. Potterat and Dr. Muth who provided me the data from this study to acquire simulations used in my paper. In this study, they collected data from 15 years of interviewing Human Immunodeficiency Virus (HIV) positive patients for at-risk partner information to elucidate network partners and their association with epidemic phase [1]. By uniquely identifying partner names by respondents, they created personal networks that generated a view of the larger networks with embedded patients and partners. In this study they included several factors during the interview such as sexual partners, injecting drug use, other sexually transmitted disease history, and local HIV/AIDs surveillance information. They then classified individuals depending on these factors. For instance, G+ in this study indicates HIV positive gay men without history of drug injection where
'G' represents Gay men. NG+ indicates HIV positive gay men with history of drug injection, in which $\mathrm{N}$ identifies individuals with drug use. Likewise, (-) and ? represents HIV negative and unknown HIV status, respectively. Additionally, they used 'F' to indicate women and ' $\mathrm{M}$ ' to illustrate heterosexual men. Interestingly, homosexual women were not included in this study because they played little to no role in HIV transmission. Of the 1100 men with HIV/AIDS for individuals whose risk factor information was available, $86 \%$ (945) were gay with 185 reported histories of injecting drug use, 12.1\% (133) reported histories of injecting drug use and heterosexuality, 1.6\% (18) classified as transfusion associated cases, and $0.2 \%$ (2) were heterosexual cases. In this study, typical member of the largest early period component was a white gay man nearly 30 years old who were associated with drug injection. This finding demonstrates how homosexual men and drug injection positively correlate in HIV/AIDS transmission. This study provides a simulation of the 250 individuals in which 156 were identified solely by HIV contact interviews while the remaining 94 people were identified from the other sources. Removal of these 94 people identified from the other sources did not alter the geometry of this network, proving how the observed geometry of HIV partners risk networks mirrors reality. Particularly, of 156 people, approximately $90 \%$

*Corresponding author: James Yeongjun Park, Department of Physics, Emory University, United States, Tel: 646-574-5498; E-mail: james.park@emory.edu

Received January 27, 2017; Accepted February 01, 2017; Published February 08, 2017

Citation: Park JY (2017) A Call to Action for More Effective Preventative Care Strategies for HIV in Men Having Sex with Men (MSM): A Combined Computational Model and Qualitative Analysis. J AIDS Clin Res 8: 661. doi: 10.4172/2155 6113.1000661

Copyright: (c) 2017 Park JY. This is an open-access article distributed under the terms of the Creative Commons Attribution License, which permits unrestricted use, distribution, and reproduction in any medium, provided the original author and source are credited. 
of these individuals were gay/bisexual men. This founding suggests that this cluster may represent an accurate network and thus reality; other studies demonstrate similar conclusions. For instance, Brenner et al. substantiates that there has been a decline in incident HIV transmissions within small cluster transmissions where the majority of onward spread of the epidemic in Quebec can be ascribed to thirty clusters [2]. In another study, Chan et al. used phylogenetic tree to determine how MSM formed $52 \%$ of clusters; they suggest that characterization of transmission dynamics among MSM reveals actively growing clusters, which should be targets for interventions [3]. These findings suggest an importance of a more delineated investigation to understand HIV/ AIDS transmission especially in these big clusters.

I was able to reproduce this image using the original data set provided by the original author of Risk network structure in the early epidemic phase of HIV transmission in Colorado Springs. The image was generated from Pajek, a program package for analysis and visualization of large networks, after realizing a planar representation for the cyclical portion of the largest component of the graph where no lines crossed. It is still the only general program that can facilitate big networks with billions of vertices without the limit. "In Pajek analysis and visualization of large networks are performed using six data types: networks; partition; vector; cluster; permutation; and hierarchy" [4]. Its intricacy enables abstraction by decomposition of a large network into smaller networks, gives useful visualization tools and promises efficient algorithms for large network analysis. Results acquired from using Pajek are usually further analyzed by specific software programs like SPSS and SAS, but I exported them and further analyzed then using $\mathrm{R}$ and Excel as SPSS and SAS are very expensive, and my coding competency was insufficient to proceed full analysis of the raw data

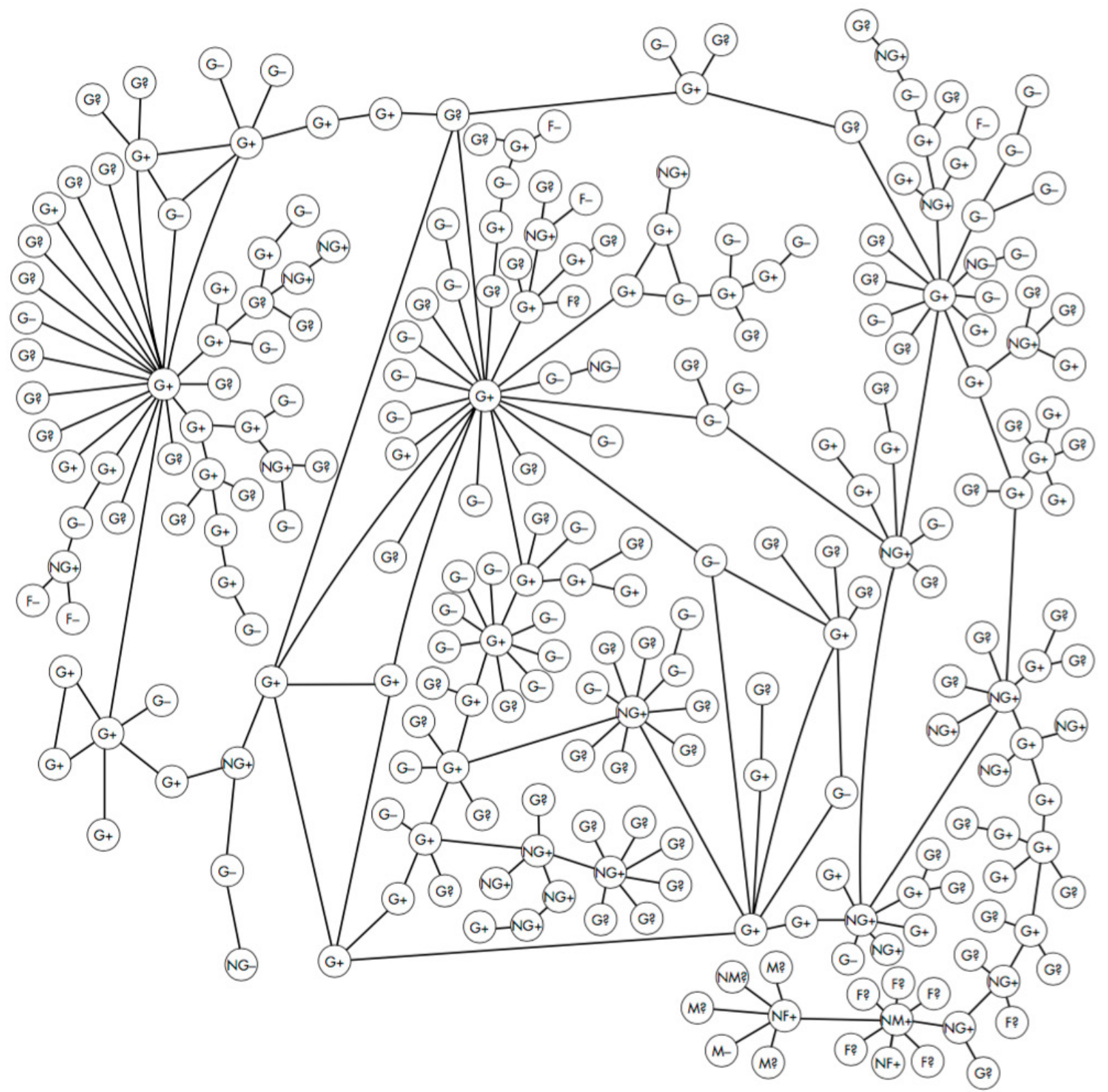

Figure 1: Cluster representing an accurate network and thus reality. 


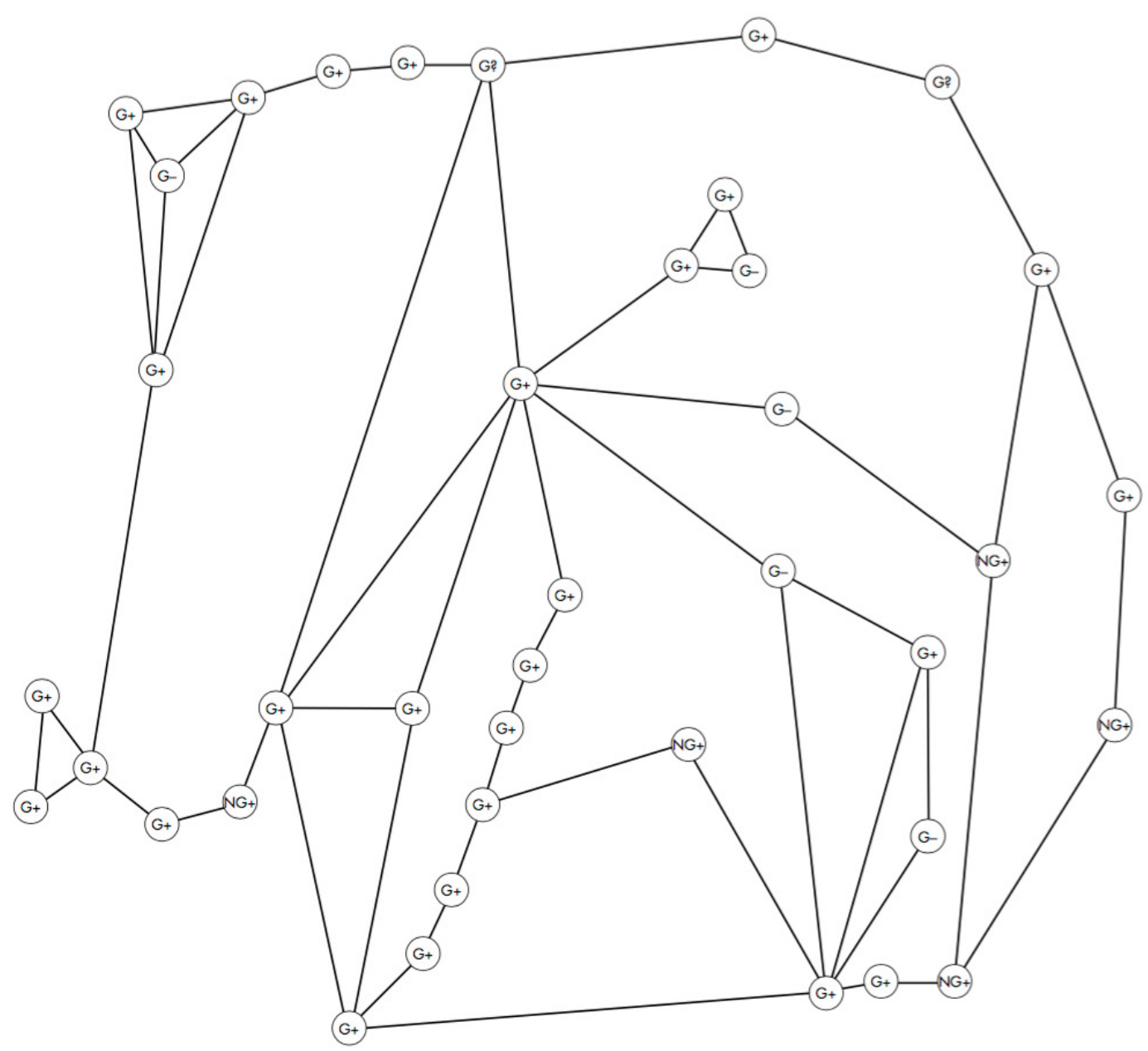

Figure 2: Cluster representing an accurate network and thus reality.

using SAS and SPSS. The file 'toPajek250.sas' is the SAS file initially used to generate hiv.250.net. 'Hiv250.net' is the Pajek file used to generate the figure where 'hiv250.xy' is the screen coordinates that prevented hiv250.net file from losing coordinates when working with Pajek (Figures 1 and 2).

\section{Introduction}

HIV had been persistent in human populations decades initial to its first discovery, but the fact that HIV was first discovered among gay men elicited the global response. In contemporary "society, these individuals and their communities continue to suffer from stigma, criminalization, discrimination, and scarceness of access to HIV services, in which homophobia continues to potentiate the epidemic" [5]. HIV epidemics among men having sex with men (MSM) are undeveloped despite advances in treatment and prevention paradigms. On both human rights and public health grounds, expansion of HIV preventative treatment and care to MSM is urgently needed. Efficacious combination prevention and treatment approaches are possible when we combine both behavioral and syndemic interventions. Antiretroviral therapy (ART) has shifted HIV/AIDS from a deadly infection to a treatable, preventable disease. In a study done by Brenner et al. ART contributed to a significant reduction ( $25 \%$ to $50 \%$ decline) in heterosexual epidemics worldwide, which raised optimism that Treatment-as-Prevention (TasP) could potentially end the pandemic [2]. In accordance with TasP, declines in new infections in countries like sub-Saharan Africa, universal declining trends in AIDS, and advances in treatment and preventative methods has provided a promising optimism of HIV pandemic control [6]. However, a paradoxical increase of HIV transmission among MSM in Western world challenges the feasibility of TasP. They found that annual numbers of HIV diagnoses among the MSM population have remained unchanged despite the proportion with viral suppression. Therefore, the continued rise of the HIV transmission suggests the need for more effective, sustainable reduction strategies. Epidemics of HIV in MSM continue to expand in varying income countries, and current treatment and prevention strategies are insufficient for this HIV spread in MSM. In the U.S, there is a continuous increase and high incidence of HIV transmission among MSM that represent $57 \%$ of new infections in 2009 in the 40 states reporting HIV infection [7]. Although diagnoses among other groups have been stably declining, Centers for Disease Control and Prevention (CDC) shows an unchanged increase in new HIV diagnoses among MSM in which similar patterns are shown in other countries aside from western countries including the States. Vosburge also found that a model based on annual HIV data since the introduction of highly antiretroviral therapy predicted that among a cohort of HIV-negative MSM, $41 \%$ would be infected by the age 40 . In contrast to trends reported in the 1990s, the burden among MSM is now increasing faster among other populations. The estimated yearly numbers of new diagnoses of HIV/AIDs among MSM in the 
33 states with named reporting increased by $11 \%$ from 2001 to 2005 while decreasing by $20 \%$ among all other people in these 33 states. This striking statistics suggests an urgent need to study the association between MSM and HIV transmission for further preventative strategies. Unlike western countries, sex between men is relatively uncommon and thus a less important route of HIV transmission in sub-Saharan Africa. In sub-Saharan Africa, the majority of HIV transmissions occur among sex between men and women. However in a high risk cohort of MSM in Coastal Kenya, $83 \%$ reported bisexaul behaviour during their follow-up and the estimated HIV incidence was as high as nine per 100 person's year. This suggests that public health interventions should target both MSM and heterosexual population to reduce transmission even in a place like Kenya where heterosexual population preponderate [8]. It has been found that MSM are more likely than others living with HIV to be in networks of transmissions where most identified were among MSM. In the Swiss national cohort, $2 / 3$ of newly diagnosed HIV infections were identified as MSM, significantly more likely than other HIV persons to be in transmission clusters. Taken together, this information suggests that HIV spread in networks of networks is efficient, consistent, and driven by high onward transmission probability in which the epidemic is otherwise in decline. Much of the work on HIV acquisition and transmission focuses on individual level risks for HIV infection, which have shown to be insufficient to explain the divergence of MSM epidemics. Scale-up of HIV preventative services for MSM is particularly difficult due to homophobia, stigma, criminalization and sub-optimum access to HIV testing and care. These factors complicate effective HIV prevention programs for MSM. Therefore, developing preventative treatment, policy and program is imperative to explain such consistent trends of HIV among MSM and then address this ongoing component of global HIV/AIDs transmission in order to achieve global control of the HIV pandemic.

Epidemics in MSM are re-emerging in all high, middle, and lowincome countries. These trends suggest an urgent need for the better HIV prevention strategies. HIV prevention is especially complicated for MSM because of the high biological risk from anal intercourse, higher frequency and types of sexual activities, little acknowledgement of male-male sex by healthcare providers and governments and syndemic, the aggregation of two or more diseases in a population in which there is some level of positive biological interaction that exacerbates the negative health effects of any or all of the diseases, challenges. The biological and network-level transmission dynamics substantiate that antiretroviral approaches decreasing probability of per-act transmission are enhanced and needed additional to behavioral and structural interventions.

\section{Methods and Results}

\section{Biomedical interventions}

Pre-Exposure Prophylaxis (PrEP) is generally accepted among societies as they are effective and translate to institutional practices. PrEP has been recognized as a potential means to maximize HIV prevention strategies. "The first successful PrEP clinical trial was conducted among MSM and showed efficacy of $44 \%$ for daily tenofovir/emtricitabine (TDF/ FTC) for prevention of HIV. Over 10 years, population coverage of $40 \%$ at risk HIV-negative MSM with PrEP is estimated to prevent approximately $25 \%$ of new HIV infections; increasing coverage to $80 \%$ results in prevention of $40 \%$ of new infections" [9]. However, many barriers incapacitate effectiveness of PrEP and actual PrEP usage has remained low since its first discovery. Specifically, awareness of PrEP has been increased among MSM; however, it has been found that PrEP messaging may not be available for those at highest risk including younger and African American males. "A September 2015, Kaiser Family Foundation survey found less than half of gay and bisexual men were aware of PrEP, and only 5\% had ever used PrEP. Among MSM at highest risk of HIV infection, knowledge of PrEP was lower and inconsistent" [10].

The current pricing structure especially in industrialized countries is a blatant impediment to a more prevalent use of this new intervention. Therefore, strategies to normalize the use of PrEP in the society may serve as the key to this conundrum [2]. New prevention strategies like the combination of tenofovir and emtricitabine (Truvada) chemoprophylaxis, oral pre-exposure prophylaxis, and increased protection of anal intercourse have efficacy for MSM suggests tangible possibilities for the ultimate achievement of control of HIV sub-epidemics in MSM. Despite proven efficacy, their high cost prevents general populations from readily seeking out these treatments. Reductions in cost of antiretroviral drugs for HIV prevention and for treatment are pivotal to the feasibility of antiretroviral approaches to HIV in MSM. To achieve measurable success in the prevention of HIV in MSM, raising awareness and funding to lower the costs of antiretroviral medications is necessary. Although antiretroviral therapy has incredible life-saving potential, it is expensive, may not cure, and can have debilitating side effects for certain individuals. Behavioural and biomedical interventions are complementary for HIV prevention in MSM. Although it is extremely crucial, biomedical interventions themselves are insufficient to reduce HIV transmission in MSM. Different strategies for PrEP and antiretroviral treatment delivery are desired in order to produce the necessary efficacy for MSM who are at the highest risk for HIV. Provided this status quo, it is necessary to make sustained changes in order to achieve the magnitude of HIV protection in MSM that may alter the course of the epidemic. Unwillingness of MSM to try PrEP can be attributed to many factors including structural issues like social stigma and criminalization. Therefore, delivery of these biomedical interventions coordinated with behavioral and structural strategies optimize the effectiveness of prevention.

\section{Behavioral interventions}

Many studies report association between injection and noninjection substance and sexual risk behavior among MSM that may result in HIV transmission. Substance use is one key factor noted in high HIV incidence rates among MSM as the rates of illicit substance use are significantly higher among MSM in comparison with the general US population and heterosexual individuals. Many factors lead to an increasing drug use. Studies have found that prevalent drug use is related to demographic and socioeconomic strategies [11]. For instance, they showed more precise correlations between community characteristics and HIV infection among individuals with injecting drug use than their individual behaviors. Bluthenthal determined that 'bad' communities like residential segregation, poverty, income inequality, high unemployment, and various other indices of social and economic disadvantage correspond to higher HIV risk behaviors and likelihood of injecting substances. It has already been established that injecting substances is directly associated with higher rates of HIV transmission because there is a blatant link between injection drug use and additional risk opportunity. However, it has also been found that non-injecting drug use also correlates with high incidence HIV rates among MSM. Out of 15 substances measured from this study, only methamphetamine use and binge drinking were consistently associated with participating in sexual risk behavior among MSM. Other researches provide some potential factors resulting in an excessive alcohol use among MSM are stigma or anxiety management, socialization to alcohol use, the 
historical bars in gay culture, and socioeconomic issues. Additionally, it has been found that $90 \%$ of men use Methamphetamine for enhanced sexual pleasure. In addition to methamphetamine, cocaine and poppers use are consistently associated with unprotected sex and thus HIV seroconversion. These demonstrate that MSM are more engaged in both injecting and non-injecting drug use, and much evidence displays that alcohol and use of drugs are key drivers of HIV infections in MSM communities worldwide. Thus, drug interventions that reduce alcohol consumption and the use of stimulants like methamphetamine can be important adjunctive approaches in MSM and could lead to a decreased HIV infection. This founding suggests substance abuse treatment is imperative for effective HIV risk-reduction interventions where MSM should be routinely screened for HIV and STIs.

In addition to these substance uses, previous behavioral interventions targeted other risky behaviors such as unprotected anal intercouse, number of sexual partners, adherence to antiretrovirals and more frequent HIV testing/screening. After the initial discovery of the viral cause of HIV, MSM reduced their numbers of sex partners and engaged in safer sex (i.e., used condoms more often) and the incidence of HIV infection drastically fell in some regions. Evidence demonstrates that condoms and pre-exposure care with antiretrovirals reduce the risk of HIV infection. Researchers analyzed the efficiency of interventions to increase dependence on antiretroviral therapy, which is preventive of suppression of HIV viral load. This adherence could be exploited as a basis for development of adherence strategies for HIV-negative MSM prescribed oral pre-exposure prophylaxis. Treating HIV positive MSM to reduce HIV transmission is biologically plausible and despite its controversy. As prevention strategies adhering to antiretroviral or prophylaxis are incorporated, clinical surveillance systems will be important to monitor to strengthen their effects. HIV testing itself is an intervention because people who are examined as HIV positive take efforts to reduce the risk of transmission to others. Many MSM are unaware of their serostatus and accurate knowledge of serostatus is crucial to reduce HIV transmission. However, any single prevention modality will not provide complete protection from HIV infection. Interventions aiming at these behaviors seemed to decrease the frequency of unprotected sex, these were not very efficient because there were only very few resources available to bring individual approaches to scale [12]. For instance, the benefits of antiretroviral treatments could be useless if MSM start engaging in risky behaviors because of perceived protection from such interventions. Therefore, even places like in North America or Europe where most substantial investment has been made in the scale-up of behavioural interventions, biomedical approaches are increasingly emphasized.

These individual level risks for HIV transmission are informative, but it has been shown that they are insufficient to explain HIV epidemics among networks of men and to explain the marked disparities in sub-epidemics of MSM where individual level risks may be most but cumulative acquisition probabilities high [5]. Interventions to increase condom use or reduce sexual partner number have shown somewhat modest reductions in self-reported outcomes from HIV-positive persons, but there were no effects on HIV incidence outcomes through these individual level interventions. Likewise, some behavioural interventions are useful in reduction of the likelihood of unprotected anal intercourse in MSM, but none decreases the incidence of new HIV infections. These findings suggest that HIV prevention for MSM must utilize approaches intervening at key points in the HIV transmission cycle to allow the provision of prevention methods specifically targeted to men's unique individual circumstances [8]. Available prevention and treatment tools are not sufficient as individual level interventions only reach insufficient levels of coverage and continue to be impeded by stigma, homophobic, discrimination and inadequate healthcare. In so being, behavioral interventions have an important role in combination with biomedical interventions [6]. In many trials, behavioral interventions for MSM reduced unprotected anal sex by $27 \%$ compared to minimal or no intervention and also reduced the proportion of men reporting any unprotected anal sex by $23 \%$. Although yielded many successes, addressing individual-level risk behaviors while neglecting contextual factors contributing to infection risk will not significantly reduce the infection. Combination of varying treatment interventions to optimize effectiveness has been advocated; therefore, it is necessary to consider other societal and structural factors that contribute to HIV transmission. Prevention methods are most likely to succeed when addressing all these major drives of HIV epidemics with primary interventions and suggest fundamental strategies supporting prevention and ethical imperatives for MSM. HIV treatment will advance when incorporating the provision of culturally competent care, the realization of the right to health, and reduced syndemics.

\section{Structural interventions}

Prevention strategies lowering biological risks including those antiretrovirals have provided promising effects for epidemic control, but are limited by discrimination, criminalization, and barriers to healthcare. In too many settings even now, MSM continue to not have access to the most basic HIV services and intervention technologies such as affordable condoms, appropriate lubricants and accessible HIV counseling and testing. The association between racial minority status and the disproportionate burden of preventable disease is a commonly observable and recognized fact in the US; however, this is also very true among LGBTQ individuals. LGBTQ persons (thus MSM) have the special healthcare needs, yet there are the barriers they face in obtaining appropriate services, which result in higher HIV transmissions. Until very recently, there was a lack of data that impeded from identifying and characterizing health disparities among MSM. It was until recently that clinicians and public health professionals realized that LGBTQ persons constitute a group that requires unique, specialized healthcare, and this finding is heightened by the emergence of the HIV epidemic. Therefore, clinicians and healthcare researchers have learned the range of existing health disparities and distinct clinic issues affecting MSM, yet they have been incapable of filling the voids remaining from previous biases towards LGBTQ persons. In a study, Wolitski et al. asserted that optimal healthcare for LGBTQ populations require access to both competent medical personnel and sensitive prevention services, which are constantly prevented by the barriers to accessing care. Wolitski provides four main issues that LGBTQ persons face in healthcare: 1) Reluctance by some LGBTQ patients to disclose sexual and gender identity when receiving medical care, 2) Insufficient numbers of providers competent in dealing with LGBTQ issues as part of the provision of medical care, 3) Structural barriers that impede access to health insurance and limit visiting and medical decisions making rights for LGBTQ persons and their partners, and 4) A lack of culturally appropriate prevention services [13]. These barriers that MSM constantly face in accessing optimal healthcare fail to reduce HIV transmissions and rather increase the infections. The provision of competent care of MSM requires training healthcare workers to recognize medical conditions that may be specific for MSM due to behavioral health concerns, distinctive practices, internal or external homophobic and community norms that often require specific approaches. The management of healthcare must have expectations for the provision of culturally competent care and support the providers to satisfy the expectation by providing resources needed. 
Historically, MSM have received inadequate and often discriminatory medical care in all environments [5]. Previous and current availability of the most basic HIV preventive cares for MSM is poor. MSM are at heightened risk of infection because of denial, persecution and refusal to include MSM within prevention programs in most parts of the world [14]. A weighted analysis substantiated that less than a third of MSM globally were tested for HIV infection or reached by any kind of HIV prevention programs; additionally, coverage of HIV prevention services for MSM is often inadequate. Research substantiated that stigma negatively affected the outcomes of many forms of stigma influencing MSM where stigma affected the provision and uptake of HIV care, treatment, and prevention services. It can also limit funding for targeted research and preventative programs. Both HIV-related stigma and MSM-related stigma discourage men from disclosing to their healthcare providers about their sex partners and clinical services information. Also, criminalization of HIV transmission and homosexual intercourse prevent gay men from having honest conversations about their risks for HIV, which then further prevent the adequate recommendations for services and commodities can be made. Health service avoidance results in MSM presenting late to healthcare with increased HIV burdens. Strong disincentives for MSM to disclose their sexuality to health practitioners result in failures for preventative screening and other comprehensive sexual healthcare. Comprehensive clinical care necessitates informed practitioners to inform their patients about logistics in which the patients can successfully avoid HIV transmission and acquisition like the provision of condoms, appropriate lubricates, and discussion of chemoprophylaxis. Providers must be able to regularly screen MSM patients for coexistent mental health issues like depression and substance use and help the patients for appropriate referrals to local services to address the concerns. Therefore, policy work to facilitate decriminalization of HIV transmission and homosexual sex is imperative. Also, given the pervasiveness of HIV and mental health concerns among MSM, integrated intervention and preventative services are needed. This continued exclusion of MSM from healthcare and social participation must change. MSM are at heightened risk for HIV infection; therefore, it is crucial that they are not excluded in order to achieve control of AIDs worldwide. The safe and effective basic prevention services for MSM including condom and safe lubricant distribution work at its optimal level only when men can safely seek services, communicate openly about sexual lives and eventually adopt available preventative cares.

Sexual and gender minorities often undergo rejection that may create internalized homophobic and low self-esteem. Explanations for the increased risk for HIV in MSM include factors that might be stemmed from homophobic experiences and societal pressures to conform to heteronormative society. MSM may experience shame and depression when they first recognize that their homosexual attraction is socially unacceptable [5]. Some studies found a higher pervasiveness of eating and body image disorders among MSM. Also, many LGBTQ persons endure the persistent stress of hiding one's sexuality or constantly facing verbal, emotional or physical abuse from their family members and communities. These may lead to the development of mental health disorders that can manifest as a result of living marginalized lives. Even though the cause of mental disorders is complicated, common factor among MSM is societal homophobia. Globally, the age of sexual debut is decreasing, and earlier conflicts emerge among MSM at times when they are most vulnerable. These conflicts are shown to lead to frequent substance abuse, transactional sex and STIs in their adolescents [14]. The association between health conditions and HIV/AIDS is known as a syndemic and the extent to which MSM experience other dangerous psychosocial health problems has often been measured by AIDs researchers as a means of explaining the distribution and consequences of HIV infection. Stall et al. demonstrated that psychosocial health problems within MSM resulted in increased vulnerability to HIV infection [15]. Syndemic conditions such as depression, substance use, violence, sexual stigma and homelessness are assessed to be directly associated with unprotected anal intercourse and thus HIV infection. In a study done by Glenn-Milo Santos, of all 3934 participants across 151 countries, $46 \%$ had experienced or knew someone who had experienced violence for being perceived as MSM and nearly a quarter used illicit substances. Depression was reported by $17.5 \%$ while high levels of sexual stigma were reported by $8.1 \%$ [14]. HIV-related stigma from healthcare providers was experienced by $16.6 \%$. This metaanalysis evinces syndemics that synergistically increase HIV risk among MSM. Every syndemic condition was significantly associated with at least one other condition, substantiating the interconnection of all these conditions. Overall, $67 \%$ of participants had one or more syndemics, $19.9 \%$ had two syndemic conditions, and $4.7 \%$ had three or more syndemics. Among the participants, the overall prevalence of UAI and HIV infection was $41 \%$ and $17.9 \%$. Greater numbers of health problems were significantly and directly associated with HIV infection and high-risk sexual practices. This study identified a linear increase in risk for UAI and HIV infection. MSM who reported a combination of depression, substance use, and violence had the greatest risk of HIV infection, demonstrating the interconnectivity of health and social concerns of MSM [16]. The high prevalence of two or more co-occurring psychosocial conditions among MSM suggests the challenges faced by marginalized communities at multiple levels. The existence of syndemics makes it harder for MSM to be benefited by standard HIV prevention programs, and this elucidates the dire need for comprehensive integrated preventive services to adequately address the broad spectrum of MSM health issues especially as HIV continues to disproportionately impact MSM worldwide. The prevalence of syndemic conditions among MSM is alarming, underscoring the need for increased attention to the psychological well-being of this group to alleviate their suffering as well as to minimize the impact of depression on self-care and associated risk behaviors. This substantiates how efficient HIV prevention strategies must have a broader development framework accounting a range of interconnected health issues. These multi-level strategies are imperative to efficiently ameliorate the health and social concerns facing MAM, improve their general well-being and ultimately curb transmissions of HIV in MSM.

\section{Discussion}

Efficiently addressing the expanding epidemics of HIV in MSM require continued research with increased resources, which will often involve policy change, strategic planning, and community engagement. To effectively reduce HIV and advance the health of MSM, a multitude of conditions must be addressed simultaneously through multi-level approaches targeting both individual and structural risk factors. Structural interventions like training healthcare workers in LGBT sensitivity, decriminalization of homosexual behavior, and reducing homophobia and stigma can serve as an essential key to solve this HIV pandemic for MSM. Globally, only MSM have been and are willing to fight anti-gay stigma, endure the risks incorporated in providing adequate services, and demand appropriate health services. MSM have 19.3-fold greater odds of transmitting HIV compared with the general population. Achieving the ultimate goal of an AIDS-less generation will only be facilitated with new and more effective approaches implemented at scale for MSM. 
Addressing individual-level risk behaviors while neglecting contextual factors contributing to infection risk will not significantly reduce the infection. Because discrimination and lack of social support are directly associated with HIV infection in MSM, it is important to have supportive families and educational systems for the healthy development of MSM, which is fundamental to the success of primary prevention. Structural factors, including legal and policy conditions, substantially influence access to HIV services for MSM. Therefore, donors and governments must support MSM and capacitate development of technological availability and provide engagement in policy making. Additionally, program planners and policy makers need more descriptive interventions and quantitative estimates of intervention effects to make informed choices regarding prevention research and further studies [17]. A recent report investigated HIV program implementation and discovered a powerful correlation between criminalization of homosexual behavior and lack of investment in services. Stigma and discrimination at many levels still remain as substantial impediments to HIV services for gay and other MSM. It is crucial to overcome barriers to prevention, treatment, and care for MSM that necessitate decriminalization of homosexual behaviors and repeal of discriminatory laws, policies, and practices influenced by homophobia, stigma, and discrimination. Reduced societal homophobia and provision of culturally competent healthcare services result in significantly improved outcomes for MSM patients. Additionally, interventions for MSM should be efficient and include condom and safe lubricant distribution, access to safe and regular confidential HIV testing and counseling, antiretroviral therapy, STI care, and access to rudimental healthcare services with dignity and equity. With all these barriers circumvented, the PrEP, ART and other biomedical interventions would be able to achieve the desired effectiveness for MSM at highest risk of HIV.

Findings from HIV literature are consistent with other studies among MSM that show a high prevalence of depression, sexual harassment, violence, alcohol use and other syndemic conditions. The deleterious effect of stressful life experience such as discrimination and stigma on physical and mental health have been systematically studied and proven to be correlated with HIV transmissions. Therefore, provision of integrated services for mental health issues and substance use provide additive advantages and synergistic benefits. This study demonstrates that coordinated biomedical, behavioral and structural interventions incorporating effective strategies can substantially decrease the incidence of HIV infection in MSM. More research is also needed to identify which behavioral strategies will be the most effective in decreasing transmission among MSM and which components are most effective in influencing these behaviors. Furthermore, research measuring the incidence of HIV and other STDs is needed.

\section{References}

1. Potterat JJ, Phillips-Plummer L, Muth SQ, Rothenberg RB, Woodhouse DE, et al. (2002) Risk network structure in the early epidemic phase of HIV transmission in Colorado Springs. Sexually Transmitted Infections 78: i159-i163.

2. Brenner BG, Ibanescu RI, Hardy I, Stephens D, Otis J, et al. (2016) Large cluster outbreaks sustain the HIV epidemic among men having sex with men (MSM) in Quebec from 2002 to 2015. AIDS.
3. Chan PA, Hogan JW, Huang A, DeLong A, Salemi M, et al. (2015) Phylogenetic investigation of a state-wide HIV-1 epidemic reveals on-going and active transmission networks among men who have sex with men. J Acquir Immune Defic Syndr 70: 428-435.

4. Mrvar A, Batagelj V (2016) Analysis and visualization of large networks with program package Pajek. Complex Adaptive Systems Modeling 4: 6.

5. Beyrer C, Andrea LW, Walker D, Johns B, Frangiscos S, et al. (2011) The global HIV epidemics among men who have sex with men. The World Bank.

6. Johnson WD, Diaz RM, Flanders WD, Goodman M, Hill AN, et al. (2008) Behavioral interventions to reduce risk for sexual transmission of HIV among men who have sex with men. Cochrane Database Syst Rev.

7. Vosburgh HW, Mansergh G, Sullivan PS, Purcell DW (2012) A review of the literature on event-level substance use and sexual risk behavior among men who have sex with men. AIDS Behav 16: 1394-1410.

8. Bezemer D, Cori A, Ratmann O, van Sighem A, Hermanides $\mathrm{HS}$, et al (2015) Dispersion of the HIV-1 epidemic in men who have sex with men in the Netherlands: A combined mathematical model and phylogenetic analysis. PLoS Med 12: e1001898.

9. Kelley CF, Kahle E, Siegler A, Sanchez T, Del Rio C, et al. (2015) Applying a PrEP continuum of care for men who have sex with men in Atlanta, Georgia. Clin Infect Dis 61: 1590-1597.

10. Hamel L, Firth J, Hoff J, Kates J, Levine S, et al. (2014) HIVIAIDS in the lives of gay and bisexual men in the United States. Kaiser Family Foundation.

11. Bluethenthal RN, Do DP, Finch B, Martinez A, Edlin BR, et al. (2007) Community characteristics associated with HIV risk among injection drug users in the San Francisco Bay area: A multilevel analysis. J Urban Health 84: 653-666.

12. Stall R, Purcell DW (2000) Intertwining epidemics: A review of research on substance use among men who have sex with men and its connection to the AIDS epidemic. AIDS Behavior 4: 181-192.

13. Wolitski RJ, Stall R, Valdiserri RO (2009) Unequal opportunity: Health disparities affecting gay and bisexual men in the United States. J Gay Lesbian Ment Health 13

14. Santos GM, Do T, Beck J, Makofane K, Arreola S, et al. (2014) Syndemic conditions associated with increased HIV risk in a global sample of men who have sex with men. Sex Transm Infect 90: 250-253.

15. Stall R, Mills TC, Williamson J, Hart T, Greenwood G, et al. (2003) Association of co-occurring psychosocial health problems and increased vulnerability to HIVIAIDS among urban men who have sex with men. Am J Public Health 93 939-942.

16. Chakrapani V, Newman PA, Shunmugam M, Logie CH, Samuel M (2017) Syndemics of depression, alcohol use and victimization, and their association with HIV-related sexual risk among men who have sex with men and transgender women in India. Glob Public Health 12: 250-265.

17. Beyrer C, Sullivan PS, Sanchez J, Dowdy D, Altman D, et al. (2012) A call to action for comprehensive HIV services for men who have sex with men. Lancet 380: 424-438. 